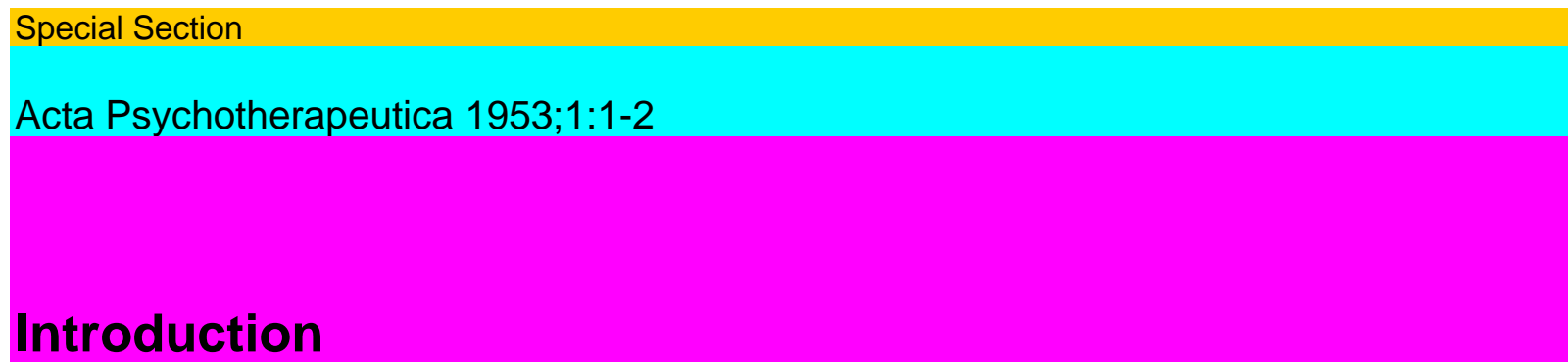

The publication of a new journal in the field of psychotherapeutics requires some justification. The editors believe that this justification is to be found in the great and growing need for further study of fundamental problems in a field in which the consideration of man as a unit must form the basis of every form of psychotherapeutic endeavour. Thorough revision is required not so much of the psychotherapeutic techniques employed as, above all, of our understanding of the basic principles underlying psychotherapeutic concepts. In our opinion the mind and body of man do not constitute two entities which can be separately considered. We look upon the patient simply as one kind of a "person in need", bis need being in the last resort mental in character. The physician who has a true sense of vocation therefore must be a psychotherapist first and foremost.

The publishers of this Journal believe that psychotherapy is essentially a form of re-education of the individual. This is accomplished in the physical sphere by removing obstacles which prevent the full development of the physical faculties. In the mental sphere, the patient is trained to let drop faulty forms of personal existence and, by being provided with alternate ways of goal attainment, is taught to accept himself as he is.

When the role of the psychotherapist is viewed in the manner described, then the course to be followed by a psychotherapeutic journal which deals at the same time with psychosomatic medicine and with the care of handicapped persons becomes clear. It aims, then, within a strictly scientific framework at doing justice to all current thought in the fields of psychotherapy, psychosomatic medicine and special education.

The Journal has been made the official organ of the International Society for the care of handicapped persons. In accordance with its international character, it will publish articles in either English, German or French, and each paper will be followed by a summary in these three languages.

CARP STOKVIS

EINLEITUNG

Die Herausgabe einer neuen Zeitschrift auf dem Gebiet der Psychotherapie bedarf einer Begründung. Diese Begründung sehen wír in dem wachsenden Be-dürfnis nach Besinnung auf die tiefsten Fragen, die auf unserem Gebiete an den Tag getreten sind, seit man zu der Einsicht gekommen ist, daß die Mensch-Betrachtung die Grundlage für jede Form psychotherapeutischen Handelns sein soil. Es hat sich erwiesen, daß nicht die Technik, sondern in erster Linie die Besinnung auf die Grundlagen unseres Therapeutseins und unserer therapeutischen Tätigkeit einer ersten grundsätzlichen Revision unterworfen werden muß. Der Mensch in seiner zweifachen Erscheinungsform, als Körper und Geist, wird wieder in der Ganzheit des Menschseins betrachtet, und der kranke Mensch er-scheint uns nur als eine Sonderform des Menschen-in-Not. Diese Not ist ihrem tiefsten Wesen nach immer Not der Seele. Der zum Arzt Berufene ist dann auch an erster Stelle Psy c1/8otherapeut.

Dem Erscheinen der neuen, der Psychothérapie gewidmeten Zeitschrift liegt der Gedanke zu Grunde, daß Psychothérapie ihrem Wesen nach eine Form des Erziehens ist, eine Erziehung des 
Menschen in seiner Körperlichkeit durch die Befreiung von Faktoren, die ihn an der Entfaltung seines körperlichen Seins L-ehindern. Sein geistiges Wesen soil erzogen werden durch die Beseitigung einer mangelhaften Seinsart, indem Kompensationsmöglichkeiten geschaffen werden, die ihn zur Ergeburg und Versöhnung mit dem eigenen So-Sein führen.

Wenn man die Aufgabe des Psychotherapeuten in dieser Weise sieht, so scheint die Gründung einer psychotherapeutischen Zeitschrift, die gleichzeitig der Psychosomatik und der Orthopädagogik (Heilpädagogik) dienen soil, ge-rechtfertigt und entschieden nicht zwecklos zu sein.

Die Zeitschrift wird auf einem streng wissenschaftlichen Niveau im Rahmen dieser Forderungen alien psychotherapeutischen, psychosomatisehen und ortho-pädagogischen Strömungen und Anschauungsweisen gerecht zu werden suchen.

\section{CARP STOKVIS}

\section{INTRODUCTION}

L'édition d'une nouvelle Revue de psychothérapie demande à être justifiée. Cette justification se trouve, a notre avis, dans le besoin accru de reflexion sur les questions de plus en plus pressantes mises en lumière par la psychothérapie, depuis qu'on a compris que Гétude de $\Gamma$ Homme doit être à la base de tous les actes psychothérapeutiques. La nécessité s'est fait sentir de soumettre à une premiere revision non pas la technique, mais la prise en consideration des bases de notre qualité de thérapeutistes et de notre activité thérapeutique. L'Homme, dans son double aspect corporel et psychique, est considéré de nouveau dans ia plenitude de son existence comme être humain; ГHomme malade ne nous apparaît que comme an cas particulier de $\Gamma$ Homme en détresse et cette détresse est au fond d'essence spirituelle. Aussi ceux qui sont appelés à î'exercice de la medicine sont-ils en premier lieu des psychothérapeutes.

L'édition de cette revue est consacrée a la psychothérapie considérée, en principe, comme forme d'éducation; education de $\Gamma$ Homme dans sa corporéité en le líbérant des facteurs qui en empêchent le développement; education sur-tout de son être spirituel, en éliminant une façon d'etre défectueuse, en suscitant des possibilités de compensation, par Гacceptation de sa propre façon d'etre et par la conciliation aussi avec soi-même. Une telle conception de la tâche du psycho-thérapeute justifie, à nos yeux, l'édition d'une revue de psychothérapie consacrée en même temps à Гorthopédagogie et à la psychosomatique. Nous nous efforcerons a nous maíntenír à un niveau strictement scientifique et à stimuler tous les courants d'opinion et toutes les farons de voir sur le terrain de la psychothérapie, de Гorthopédagogie et de la psychosomatique.

\section{CARP STOKVIS}

